\title{
Variáveis fisiológicas de búfalas leiteiras criadas sob sombreamento em sistemas silvipastoris
}

\author{
Alexandre Rossetto Garcia( ${ }^{(1)}$, Leonardo Brandão Matos(2), José de Brito Lourenço Júnior(3), \\ Benjamim de Souza Nahúm ${ }^{(1)}$, Cláudio Vieira de Araújo( ${ }^{(4)}$ e Alessandra Ximenes Santos ${ }^{(5)}$
}

\begin{abstract}
(1)Embrapa Amazônia Oriental, Travessa Dr. Enéas Pinheiro,s/no, Caixa Postal 48, CEP66095-100 Belém, PA. E-mail:argarcia@cpatu.embrapa.br, nahum@cpatu.embrapa.br (2)Agência de Defesa Agropecuária do Estado do Pará, Travessa Piedade, № 651, CEP 66053-210 Belém, PA. E-mail: Ibmatos@gmail.com (3)Universidade do Estado do Pará, Travessa Dr. Enéas Pinheiro, oo 2.626, CEP 66095-100 Belém, PA. E-mail:joselourencojr@yahoo.com.br ${ }^{(4)}$ UniversidadeFederal do Mato Grosso,AvenidaAlexandre Ferronato, no1.200, CEP78550-000 Sinop, MT. E-mail: araujocv@bol.com.br ${ }^{(5)}$ Universidade Federal do Pará, Rua Augusto Correa, oo 01, CEP 66075-110 Belém, PA. E-mail: alessandra.ximenes@gmail.com
\end{abstract}

Resumo - O objetivo deste trabalho foi avaliar o uso de sistemas silvipastoris como ferramenta de manejo para manter as características fisiológicas de búfalas leiteiras e promover mais altos níveis de conforto térmico. Foram avaliadas 56 fêmeas adultas (79 $\pm 44,12$ meses; $575 \pm 92,90 \mathrm{~kg}): 30$ em sistema silvipastoril sem sombra útil e 26 em sistema silvipastoril com 19,9\% de sombra útil. Foram mensuradas semanalmente: frequência cardíaca e respiratória, temperatura retal e índice de conforto animal. As médias foram comparadas por análise de variância e as variáveis meteorológicas e fisiológicas foram correlacionadas pelo método de Pearson. O sombreamento diminuiu significativamente a frequência cardíaca e a temperatura retal. Em 71,4\% das observações, os animais mantidos em sistema silvipastoril com sombreamento apresentaram índices de conforto próximos ao ideal. O sombreamento mantém os parâmetros fisiológicos de búfalas leiteiras mais próximos da normalidade e melhora o índice de conforto animal. A adoção de sistemas silvipastoris na produção de bubalinos, na região tropical, pode evitar gastos energéticos com termólise.

Termos para indexação: Bubalus bubalis, Amazônia, bioclimatologia, conforto animal, ecofisiologia, sistema de pastejo.

\section{Physiological features of dairy buffaloes raised under shade in silvipastural systems}

\begin{abstract}
The objective of this work was to evaluate the use of silvipastural systems as a management option to maintain physiological features of dairy buffaloes and to provide higher levels of thermal comfort. Fifty-six adult females ( $79 \pm 44.12$ months; $575 \pm 92.90 \mathrm{~kg}$ ) were evaluated: 30 in an unshaded silvipastural system and 26 in a silvipastural system with $19.9 \%$ of shaded area. Heart and respiratory rates, rectal temperature and animal comfort index were evaluated weekly. Means were submitted to analysis of variance, and meteorological and physiological data were correlated by Pearson's method. Shading significantly reduced heart rate and rectal temperature. In $71.4 \%$ of the observations, the animals kept in the silvipastural system with shaded area showed thermal comfort index closer to the ideal level. Shading maintains the physiological parameters of dairy buffaloes closer to normal, and improves the animal comfort index. The adoption of silvipastural systems for buffalo production in tropical areas can avoid energy loss due to thermolysis.
\end{abstract}

Index terms: Bubalus bubalis, Amazon, bioclimatology, animal comfort, ecophysiology, pasture system.

\section{Introdução}

O búfalo doméstico (Bubalus bubalis L.) destaca-se pela rusticidade, e é comumente explorado para trabalho e para produção de leite e carne. Atualmente, $62,8 \%$ do rebanho bubalino brasileiro está na região Norte, principalmente nos estados do Pará e do Amapá (56,18\%) (Instituto Brasileiro de Geografia e Estatística, 2010). Contudo, o calor é um dos principais fatores restritivos à produção nos trópicos (Garcia, 2006), pois o estresse térmico leva à mudança drástica nas funções biológicas dos animais (Marai \& Haeeb, 2010), com prejuízos para seu desenvolvimento, produção, reprodução (Dobson et al., 2001), bem como para capacidade de trabalho (Ablas et al., 2007). No caso de animais mantidos exclusivamente a pasto, o problema é ainda mais sério, por não terem acesso a instalações cobertas, ao menos durante as horas mais quentes do dia (Silva, 2006).

Pesq. agropec. bras., Brasília, v.46, n.10, p.1409-1414, out. 2011 
Nos sistemas silvipastoris (SSP), essências florestais são incorporadas às pastagens para integrar o manejo $\mathrm{e}$ incrementar a produtividade por unidade de área, com interações dos componentes em diferentes magnitudes. Os sistemas silvipastoris aumentam a biodiversidade em regiões alteradas pelo uso inapropriado do solo, auxiliam a preservação de nascentes e mananciais (Dias et al., 2008), conferem maior conforto aos animais (Leme et al., 2005). Por aumentar a produtividade em bovinos (Mahecha et al., 2008; Garcia et al., 2009), acredita-se que os SSP também possam reduzir os efeitos negativos do clima tropical sobre bubalinos, especialmente na Amazônia, onde os índices de temperatura e umidade são elevados praticamente $o$ ano inteiro. Entretanto, ainda são escassas as pesquisas sobre ecofisiologia em bubalinos, sobretudo quanto ao manejo do ambiente para elevar o conforto e, consequentemente, a eficiência produtiva.

O objetivo deste trabalho foi avaliar o uso de sistemas silvipastoris como ferramenta de manejo para proporcionar maior conforto térmico a búfalas leiteiras.

\section{Material e Métodos}

O experimento foi conduzido em Belém, PA, de abril a novembro de 2006, em área experimental da Embrapa Amazônia Oriental, na Unidade de Pesquisa Animal Senador Álvaro Adolpho ( $\left.{ }^{\circ} 25^{\prime} \mathrm{S}, 48^{\circ} 26^{\prime} \mathrm{W}\right)$. O clima da região, conforme Köppen, é do tipo Afi, com precipitação pluvial média anual de $3.248,1 \mathrm{~mm}$, temperatura anual média de $27,1^{\circ} \mathrm{C}$, umidade relativa do ar de $82 \%$ e insolação anual de $2.387,9$ horas (Pacheco \& Bastos, 2007), com estação chuvosa mais pronunciada de abril a junho, e menos pronunciada de setembro a novembro.

Foram utilizados dois sistemas pastoris, que compuseram os grupos experimentais. O primeiro, sem sombra (SS), era composto de 11,4 ha de pastagem de quicuio-da-amazônia [Urochloa humidicola (Rendle) Morrone \& Zuloaga], com animais em pastejo rotacionado intensivo. Ao longo das cercas perimetrais e divisórias desse sistema, havia acácia mangium, Racosperma mangium (Willd.) Pedley, plantada a cada $4 \mathrm{~m}$ e protegida por cercas eletrificadas, em densidade de 44 árvores por hectare. À época da realização da pesquisa, as árvores apresentavam altura média de 0,70 $\mathrm{m}$ e não disponibilizavam sombra útil para os animais. Os animais mantidos nesse sistema receberam mineralização em cocho coberto, e água à vontade, em centro de manejo.

O segundo sistema pastoril, com sombra (CS), apresentava área de 5,4 ha, com pastagem formada por grama-estrela (Cynodon nlemfuensis Vanderyst) e animais em sistema de pastejo rotacionado intensivo. Ao longo das cercas divisórias e perimetrais, a cada $4 \mathrm{~m}$, havia árvores de mogno africano (Khaya ivorensis A. Chev.) e nim indiano (Azadirachta indica A. Juss.), com seis anos e 12 e $7 \mathrm{~m}$ de altura média, respectivamente, e diâmetro à altura do peito de 19,75 e $10,53 \mathrm{~cm}$, respectivamente. O estande de mogno africano e nim indiano era de 42 e 35 árvores por hectare, respectivamente, protegidas por cercas eletrificadas. A área de sombra útil aos animais era de 19,9\%. Os animais mantidos neste sistema também receberam mineralização em cocho coberto, e água à vontade, em centro de manejo.

Foram utilizadas 56 fêmeas leiteiras adultas, mestiças, das raças Murrah e Mediterrâneo (79 $\pm 44,12$ meses e $575 \pm 92,90 \mathrm{~kg}$ ), com escore de condição corporal de $3,2 \pm 0,6$ (escala de 1 a 5). Os animais foram distribuídos nos grupos em delineamento inteiramente casualizado, tendo-se respeitado a capacidade de suporte das áreas experimentais, com 30 animais no grupo SS e 26 animais no CS. Semanalmente, foram registradas frequência cardíaca (FC), frequência respiratória (FR) e temperatura retal (TR) dos animais, conforme descrito por Moraes Júnior et al. (2010). Assim, foram realizadas 14 repetições de cada variável por animal, em cada época do ano. As coletas de dados foram realizadas no turno da manhã, com início às $9 \mathrm{~h}$ e horário médio de coleta às $10 \mathrm{~h} 04 \mathrm{~min}$. Após as mensurações, o índice de conforto animal (ICA) dos animais, em ambos os sistemas, foi calculado de acordo com o teste de Benezra (ICA $=\mathrm{TR} / 38,33+\mathrm{FR} / 23$ ), indicado para avaliação do conforto de animais criados nos trópicos (Benezra, 1954).

Os dados meteorológicos (temperatura do ar, umidade relativa do ar, precipitação pluvial, velocidade do vento, brilho e radiação solar) foram registrados na estação meteorológica da Embrapa Amazônia Oriental, localizada a $1,5 \mathrm{~km}$ do experimento. $\mathrm{O}$ cálculo do índice de temperatura e umidade (ITU) foi realizado conforme descrito por Koga et al. (2004).

Para análise estatística das variáveis fisiológicas e do ICA, foi realizada a transformação de dados com finalidade de atender às pressuposições da análise de variância. As médias foram comparadas 
a 5\% de probabilidade. As variáveis meteorológicas e fisiológicas foram correlacionadas ao ICA, pelo método de Pearson, ao computar-se o coeficiente de correlação simples (r) entre as variáveis e o índice, com o teste $\mathrm{t}$, a $1 \%$ de probabilidade. Os resultados foram analisados pelo SAS, versão 9.1.3 (SAS Institute, 2002/2003).

\section{Resultados e Discussão}

A temperatura média do ar apresentou valores de $28,4 \pm 1,52^{\circ} \mathrm{C}$ no período chuvoso mais pronunciado, e aumentaram para $29,7 \pm 1,22^{\circ} \mathrm{C}$ no menos pronunciado. A precipitação pluvial acumulada no período menos chuvoso foi de $374,3 \mathrm{~mm}$, enquanto no mais chuvoso, foi de $845,4 \mathrm{~mm}$. Como esperado, a umidade relativa do ar foi mais elevada no período mais chuvoso $(77,4 \pm 11,53 \%)$, em comparação ao menos chuvoso $(69,1 \pm 12,45 \%)$. Os valores do ITU variaram entre 75,0 e 81,0 no período mais chuvoso e entre 75,8 e 82,0 no menos chuvoso, com valores mais elevados no período menos chuvoso (Figura 1). As consições meteorológicas observadas seguiram padrão semelhante às médias históricas entre 1967 e 1996, para o local do experimento (Pacheco \& Bastos, 2007). A caracterização do ambiente demonstra o desafio enfrentado pelos animais para se manterem saudáveis e produtivos na Amazônia, pois o ITU ideal

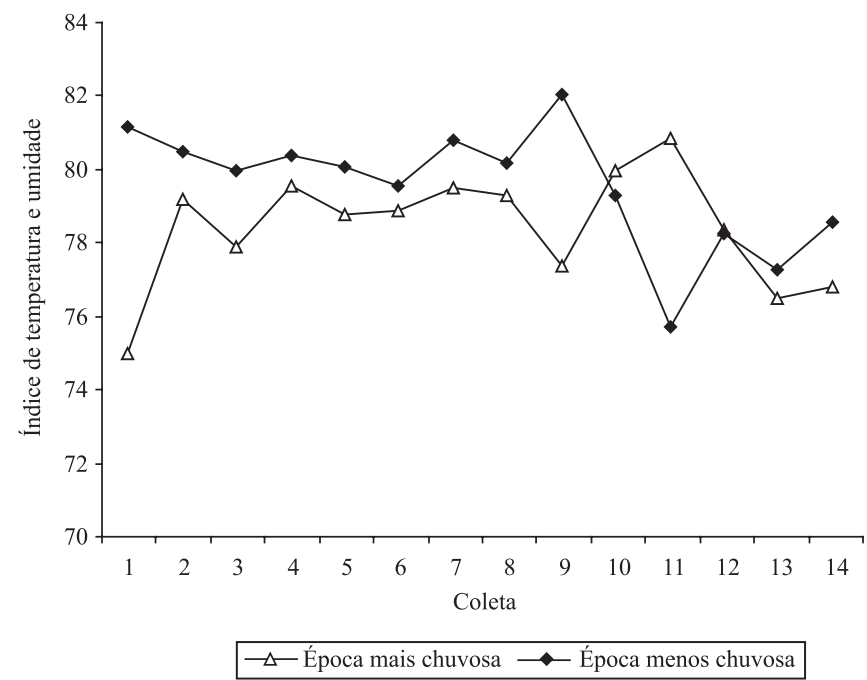

Figura 1. Índice de temperatura e umidade (ITU) registrados em experimento com sistemas silvipastoris, em Belém, PA, em 2006. Período mais chuvoso, de abril a junho; e período menos chuvoso, de setembro a novembro. para produção de bubalinos deve estar abaixo de 74,0 (Somparn et al., 2004).

Quanto aos sistemas silvipastoris usados e aos períodos estudados, a FC foi significativamente menor
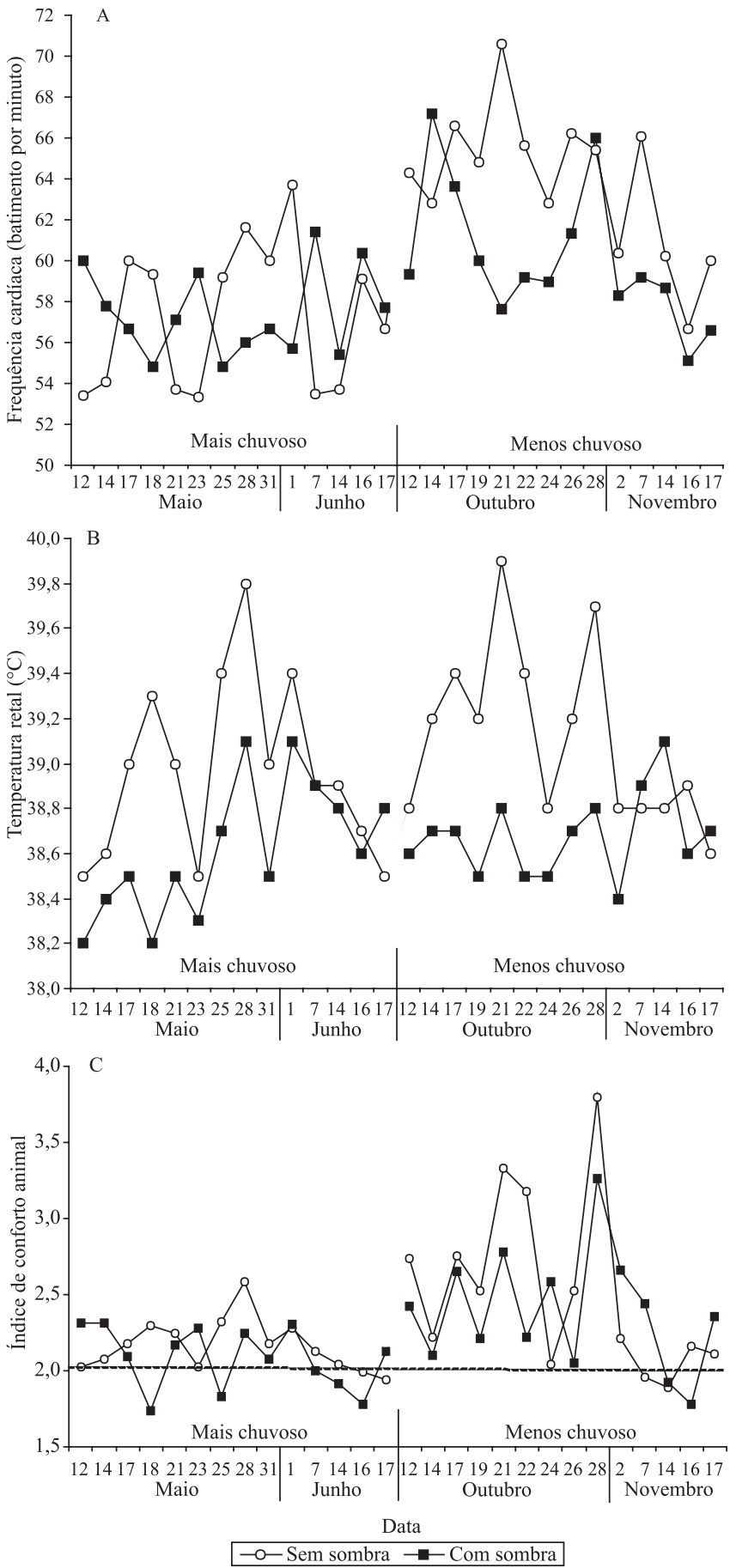

Figura 2. Frequência cardíaca (A), temperatura retal (B) e índice de conforto animal (C) de búfalas mantidas em sistemas silvipastoris na Amazônia Oriental sem sombra, e com sombra (19,9\% de área sombreada) nos piquetes, em 2006. 
nos animais do grupo CS (Figura $2 \mathrm{~A}$ ), especialmente no período menos chuvoso (Tabela 1). O sombreamento disponível protegeu os animais da incidência direta da radiação solar, diminuiu o ganho de calor ambiental e, consequentemente, a intensidade da termólise, que depende parcialmente do débito cardíaco. No período com menos chuva, quando a nebulosidade é menor e a radiação solar mais intensa, observou-se comportamento taquicárdico com maior frequência nos animais criados sem acesso à sombra.

$\mathrm{O}$ resultado observado foi ligeiramente inferior aos relatados anteriormente para bubalinos, em Belém (Castro, 2005) e na Ilha de Marajó (Lourenço Júnior, 1998), com machos de sobreano. Lourenço Júnior et al. (2006) constataram que, em temperaturas do ar mais amenas, a FC de búfalos adultos é menor e aumenta quando a temperatura média do ar ultrapassa $26,5^{\circ} \mathrm{C}$.

A FR foi mais elevada durante o período menos chuvoso, tanto nos animais manejados no SS, quanto no CS (Tabela 1). Essa elevação está diretamente relacionada à variação da temperatura ambiente e do ITU, superiores em praticamente todo o período menos chuvoso. Os búfalos usam a respiração como uma importante forma para dissipação de calor corpóreo, e sua taxa respiratória aumenta à medida que a temperatura ambiente se eleva (Das et al., 1999; Castro et al., 2008).

Embora não significativamente, os valores de TR foram inferiores nos animais criados com sombra, em ambos os períodos (Figura 2 B). O perfil de TR dos animais criados sem acesso à sombra foi mais elevado, principalmente no período menos chuvoso. Maiores valores de TR indicam maior acúmulo de calor corpóreo, com maior demanda de energia para termólise e, consequentemente, menos energia disponível para a produção, o que ocasiona baixo desempenho dos animais. Contudo, a média de TR dos animais mantidos nos sistemas CS e SS foi praticamente idêntica nos dois períodos (Tabela 1). Resultados semelhantes foram encontrados em Belém, PA, por Castro (2005), ao observar que a temperatura retal dos bubalinos variou pouco ao longo do ano, com menores valores em janeiro e fevereiro e maiores em maio, no período mais chuvoso. Lourenço Júnior (1998) obteve, na Ilha de Marajó, valores que variaram de $38,7^{\circ} \mathrm{C}$, no período chuvoso, a $39,2^{\circ} \mathrm{C}$ no período seco.

As médias do ICA observadas durante o período experimental variaram entre 1,7 e 3,8 (Figura 2 C). Em $71,4 \%$ das observações, os animais criados no sistema CS tiveram ICA mais próximo a 2,0. Segundo Castro (2005), o conforto animal está associado a melhor desempenho produtivo em búfalos para corte, pois quando o ICA apresenta valores próximos a 2,0, considerado como o mínimo para conforto animal nas regiões tropicais (Benezra, 1954), há maior ganho de peso e melhor desempenho produtivo. Os valores encontrados indicam superioridade de conforto proporcionada pelo sistema sombreado. Os dados obtidos por Lourenço Júnior (1998), para bubalinos criados na Ilha do Marajó, com índices de menor conforto térmico em outubro e novembro, diferem dos do presente trabalho, para bubalinos com acesso ao sombreamento, que mantiveram seus ICA muito próximos ao ideal, apesar do intenso calor e da radiação solar incidentes nesses meses em Belém (Pacheco \& Bastos, 2007).

O ICA apresentou correlação moderada, positiva e significativa com a temperatura média do ar (Tabela 2), o que corrobora as observações de Lourenço Júnior (1998). Guimarães et al. (2001) constataram que elevações na temperatura média do ar causam aumento na temperatura retal, a qual é parte da equação para o cálculo do ICA. A umidade relativa do ar apresentou correlação moderada, negativa e altamente significativa com o ICA, o que também confirma os resultados obtidos por Lourenço Júnior (1998). Correlações positivas, de média intensidade e altamente significativas foram observadas entre o ICA e o brilho e a radiação solar.

Tabela 1. Média \pm desvio-padrão das frequências cardíaca (FC) e respiratória (FR) e da temperatura retal (TR) de búfalas mantidas em sistemas silvipastoris com e sem sobra, em dois regimes de chuvas na Amazônia Oriental ${ }^{(1)}$.

\begin{tabular}{|c|c|c|c|c|c|c|}
\hline \multirow[t]{3}{*}{ Período } & \multicolumn{3}{|c|}{ Sem sombra } & \multicolumn{3}{|c|}{ Com sombra } \\
\hline & FC & FR & TR & FC & FR & TR \\
\hline & \multicolumn{2}{|c|}{------ (movimentos por min) ------ } & $\left({ }^{\circ} \mathrm{C}\right)$ & \multicolumn{2}{|c|}{----- (movimentos por min) ------ } & $\left({ }^{\circ} \mathrm{C}\right)$ \\
\hline Mais chuvoso & $57,26 \pm 8,35 \mathrm{Aa}$ & $26,43 \pm 11,82 \mathrm{Aa}$ & $38,94 \pm 0,56 \mathrm{Aa}$ & $57,44 \pm 8,13 \mathrm{Ab}$ & $24,79 \pm 8,78 \mathrm{Aa}$ & $38,62 \pm 0,48 \mathrm{Aa}$ \\
\hline Menos chuvoso & $64,43 \pm 8,26 \mathrm{Bb}$ & $34,79 \pm 18,15 \mathrm{Ba}$ & $39,11 \pm 0,48 \mathrm{Aa}$ & $60,20 \pm 6,52 \mathrm{Ba}$ & $34,79 \pm 18,24 \mathrm{Ba}$ & $38,68 \pm 0,32 \mathrm{Aa}$ \\
\hline
\end{tabular}

${ }^{(1)}$ Médias seguidas de letras iguais, maiúsculas entre períodos e minúsculas entre sistemas silvipastoris, não diferem entre si pelo teste $t$, a $1 \%$ de probabilidade. Na análise estatística, os valores foram transformados para escala logarítmica. 
Tabela 2. Coeficientes de correlação de Pearson entre o índice de conforto animal (ICA) de fêmeas bubalinas, e variáveis fisiológicas e ambientais ${ }^{(1)}$.

\begin{tabular}{ccccccc}
\hline & $\mathrm{T}_{\text {med }}$ & UR & VV & PP & Brilho & Radiação \\
\hline ICA & $0,49^{* *}$ & $-0,34^{* *}$ & $0,06^{\text {ns }}$ & $-0,07^{\text {ns }}$ & $0,30^{* *}$ & $0,31^{* *}$ \\
\hline
\end{tabular}

${ }^{(1)} \mathrm{T}_{\text {méd, }}$, temperatura média do ar; UR, umidade relativa do ar; $\mathrm{VV}$, velocidade do vento; PP, precipitação pluvial; Brilho, brilho solar; Radiação, radiação

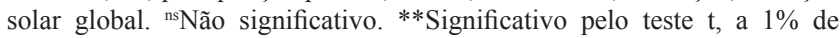
probabilidade.

Estes resultados foram semelhantes aos encontrados por Lourenço Júnior (1998) e Castro (2005), pois a radiação solar age de forma danosa, principalmente em animais de pele escura, que apresentam proteção satisfatória contra a radiação ultravioleta, mas absorvem maior quantidade de energia térmica, o que limita sua capacidade de eliminar calor e contribui para o estresse térmico (Silva et al., 2001). Além disso, animais de pele escura não estão isentos dos efeitos deletérios crônicos ou agudos da radiação ultravioleta sobre os componentes oculares (Oliveira et al., 2001), produtivos e reprodutivos (Marai \& Haeeb, 2010), o que torna o sombreamento elemento importante na manutenção da saúde dos bubalinos criados a campo na região tropical.

O nível de sombreamento das pastagens $(19,9 \%)$ foi superior a $12 \%$, valor considerado como sendo de alta disponibilidade de sombra para animais de produção, conforme Paranhos da Costa \& Cromberg (1997). É possível que, por esse motivo, o sombreamento tenha sido tão positivo nas variáveis fisiológicas e de conforto nos bubalinos monitorados, e que, por isso, seja alternativa viável para manutenção dos animais em homeostase, o que normalmente acontece somente quando os búfalos têm acesso a ambientes com água para imersão (Ablas et al., 2007). Como as adversidades do clima e das mudanças climáticas são grandes desafios que o Brasil deve superar para expandir e modernizar seu sistema de produção agropecuária (Tollefson, 2010), o desenvolvimento de novos estudos sobre a integração entre agricultura, silvicultura e produção animal faz-se necessário.

\section{Conclusões}

1. O sistema silvipastoril, por disponibilizar sombra útil nas pastagens, proporciona manutenção dos parâmetros fisiológicos de búfalas leiteiras mais próximos à normalidade.
2. O sombreamento das pastagens é eficiente para amenizar os efeitos do clima tropical quente e úmido sobre os animais e melhorar seu índice de conforto térmico, independentemente da época do ano.

\section{Agradecimentos}

À Embrapa Amazônia Oriental e ao Banco da Amazônia, pelo apoio financeiro.

\section{Referências}

ABLAS, D.S.; TITTO, E.A.L.; PEREIRA, A.M.F.; TITTO, C.G.; LEME, T.M.C. Comportamento de bubalinos a pasto frente à disponibilidade de sombra e água para imersão. Ciência Animal Brasileira, v.8, p.167-175, 2007.

BENEZRA, M.V. A new index measuring the adaptability of cattle to tropical conditions. Journal of Animal Science, v.13, p.1015, 1954.

CASTRO, A.C. Avaliação de sistema silvipastoril através do desempenho produtivo de búfalos manejados nas condições climáticas de Belém, Pará. 2005. 91p. Dissertação (Mestrado) Universidade Federal do Pará, Belém.

CASTRO, A.C.; LOURENÇO JÚNIOR, J.B.; SANTOS, N.F.A.; MONTEIRO, E.M.M.; AVIZ, M.A.B.; GARCIA, A.R. Sistema silvipastoril na Amazônia: ferramenta para elevar o desempenho produtivo de búfalos. Ciência Rural, v.38, p.2395-2402, 2008.

DAS, S.K.; UPADHYAY, R.C.; MADAN, M.L. Heat stress in Murrah buffalo calves. Livestock Production Science, v.61, p.71-78, 1999.

DIAS, P.F.; SOUTO, S.M.; AZEVEDO, B.C.; VIEIRA, M.S.; COLOMBARI, A.A.; DIAS, J.; FRANCO, A.A. Estabelecimento de leguminosas arbóreas em pastos de capim-marandu e tanzânia. Pesquisa Agropecuária Brasileira, v.43, p.1413-1419, 2008.

DOBSON, H.; TEBBLE, J.E.; SMITH, R.F.; WARD, W.R. Is stress really all that important? Theriogenology, v.55, p.65-73, 2001.

GARCIA, A.R. Influência de fatores ambientais sobre as características reprodutivas de búfalos do rio (Bubalus bubalis). Revista de Ciências Agrárias, n.45, p.1-13, 2006. Suplemento.

GARCIA, A.R.; ALVAREZ, W.F.M.; COSTA, N.A.; NAHÚM, B.S.; QUINZEIRO NETO, T.; CASTRO, S.R.S. Avaliação do desempenho de bovinos de corte criados em sistemas silvipastoris no Estado do Pará. Amazônia Ciência e Desenvolvimento, v.4, p.51-62, 2009.

GUIMARÃES, C.M.C.; FALCO, J.E.; TITTO, E.A.L.; FRANZOLIN NETO, R.; MUNIZ, J.A. Termorregulação em bubalinos submetidos a duas temperaturas de ar e duas proporções de volumoso: concentrado. Ciência e Agrotecnologia, v.25, p.437-443, 2001.

INSTITUTO BRASILEIRO DE GEOGRAFIA E ESTATÍSTICA. Efetivo dos rebanhos por tipo de rebanho - pesquisa pecuária municipal: relatório do ano de 2009. Rio de Janeiro: IBGE, 2010. 
Disponível em: <http://www.sidra.ibge.gov.br/bda/tabela/protabl. asp? $=73 \& z=t \& o=23 \& \mathrm{i}=\mathrm{P}>$. Acesso em: $23 \mathrm{fev} .2011$.

KOGA, A.; SUGIYAMA, M.; DEL BARRIO, A.N.; LAPITAN, R.M.; ARENDA, B.R.; ROBLES, A.Y.; CRUZ, L.C.; KANAI, Y. Comparison of the thermoregulatory response of buffaloes and tropical cattle, using fluctuations in rectal temperature, skin temperature and haematocrit as an index. Journal of Agricultural Science, v.142, p.351-355, 2004.

LEME, T.M.S.P.; PIRES, M.F.A.; VERNEQUE, R.S.; ALVIM, M.J.; AROEIRA, L.J.M. Comportamento de vacas mestiças Holandês $\mathrm{x}$ Zebu, em pastagem de Brachiaria decumbens em sistema silvipastoril. Ciência e Agrotecnologia, v.29, p.668-675, 2005.

LOURENÇO JÚNIOR, J.B. Variáveis produtivas, fisiológicas e de comportamento de zebuínos e bubalinos e fatores do ambiente físico em pastagem cultivada da ilha de Marajó. 1998. 159p. Tese (Doutorado) - Universidade Federal do Pará, Belém.

LOURENÇO JÚNIOR, J.B.; CASTRO, A.C.; DANTAS, J.A.S.; SANTOS, N.F.A.; ALVES, O.S.; MONTEIRO, E.M.M. Efeitos das variáveis climáticas sobre a fisiologia de bubalinos criados em sistema silvipastoril, em Belém, Pará. In: CONGRESSO BRASILEIRO DE BIOMETEOROLOGIA - MUDANÇAS CLIMÁTICAS: IMPACTO SOBRE HOMEM, PLANTAS E ANIMAIS, 4., 2006, Ribeirão Preto. Anais. Ribeirão Preto: SBBIOMET, 2006. p.1-5.

MAHECHA, L.; ANGULO, J.; SALAZAR, B.; CERÓN, M.; GALLO, J.; MOLINA, C.H.; MOLINA, E.J.; SUÁREZ, J.F.; LOPERA, J.J.; OLIVERA, M. Supplementation with bypass fat in silvopastoral systems diminishes the ratio of milk saturated/ unsaturated fatty acids. Tropical Animal Health and Production, v.40, p.209-216, 2008.

MARAI, I.F.M.; HAEEB, A.A.M. Buffalo's biological functions as affected by heat stress - a review. Livestock Science, v.127, p.89-109, 2010.
MORAES JÚNIOR, R.J.; GARCIA, A.R.; SANTOS, N.F.A.; NAHÚM, B.S.; LOURENÇO JÚNIOR, J.B.; ARAÚJO, C.V.; COSTA, N.A. Conforto ambiental de bezerros bubalinos (Bubalus bubalis Linnaeus, 1758) em sistemas silvipastoris na Amazônia Oriental. Acta Amazonica, v.40, p.629-640, 2010.

OLIVEIRA P.R.; OLIVEIRA, A.C.; OLIVEIRA, F.C. A radiação ultravioleta e as lentes fotocrômicas. Arquivos Brasileiros de Oftalmologia, v.64, p.163-655, 2001.

PACHECO, N.A.; BASTOS, T.X. Boletim agrometeorológico 2006 - Belém, Pará. Belém: Embrapa Amazônia Oriental, 2007, 34p. (Embrapa Amazônia Oriental. Documentos, 300).

PARANHOS DA COSTA, M.J.R.; CROMBERG, V.U. Alguns aspectos a serem considerados para melhorar o bem-estar de animais em sistema de pastejo rotacionado. In: PEIXOTO, A.M.; MOURA, J.C.; FARIA, V.C. Fundamentos do pastejo rotacionado. Piracicaba: FEALQ, 1997. p.273-296.

SAS INSTITUTE. Statistical analysis system: user's guide. Version 9.1.3. Cary: SAS Institute, 2002/2003.

SILVA, R.G. Predição da configuração de sombras de árvores em pastagens para bovinos. Engenharia Agrícola, v.26, p.268-281, 2006.

SILVA, R.G.; LA SCALA JÚNIOR, N.; POCAY, P.L.B. Transmissão de radiação ultravioleta através do pelame e da epiderme de bovinos. Revista Brasileira de Zootecnia, v.30, p.1939-1947, 2001.

SOMPARN, P.; GIBB, M. J.; MARKVICHITR, K.; CHAIYABUTR, N.; THUMMABOOD, S.; VAJRABUKKA, C. Analysis of climatic risk for cattle and buffalo production in northeast Thailand. International Journal of Biometeorology, v.49, p.59-64, 2004

TOLlEFSON, J. The global farm. Nature, v.466, p.554-556, 2010.

Recebido em 31 de julho de 2010 e aprovado em 22 de julho de 2011 